\title{
COMPARATIVE ANALYSIS OF THE PHYSICAL AND MECHANICAL PROPERTIES OF KRAFT PAPER AND WATERCOLOR PAPER
}

\author{
Anling $\mathrm{Li}^{1,2, \mathrm{~s}}$ \\ https://orcid.org/0000-0002-9426-9830 \\ Yameng Shi ${ }^{2}$ \\ https://orcid.org/0000-0002-5778-3347 \\ Jiaqi Zhang ${ }^{2}$ \\ https://orcid.org/0000-0002-9625-1749 \\ Yong Zhang ${ }^{2}$ \\ https://orcid.org/0000-0003-0443-2997
}

\begin{abstract}
In order to study the feasibility of kraft paper as watercolor paper, the wetting properties, mechanical properties and friction properties of kraft paper and watercolor paper were compared before and after aging. The aging test was carried out on kraft paper and the watercolor paper by aging chamber, the wetting properties of distilled water and three watercolor solutions before and after aging of kraft paper and watercolor paper were compared, tensile tear test of paper on the tensile testing machine, the fracture changes of paper were analyzed by scanning electron microscope, the friction tester performs the paper friction test. The results show that both kinds of paper are amorphous, watercolor paper was showed hydrophobicity before and after the aging, the contact angle was maintained at about $119,09^{\circ} \pm 5^{\circ}$ in four different solutions, unaged kraft paper exhibited hydrophilicity, the aged kraft paper showed hydrophobicity with a contact angle of $92,52^{\circ} \pm 1^{\circ}$, the aged kraft paper shows better performance in both tensile and tearing aspects and tensile strength is 20 MPa higher than watercolor paper, the surface roughness of kraft paper is higher than watercolor paper. The experiment is helpful for the market to consider whether the cheaper kraft paper replaces the more expensive watercolor paper in some aspects and provides some scientific reference value for future research.
\end{abstract}

Keywords: Contact angle, kraft paper, tear strength, tensile strength, watercolor paper, wear resistance.

\section{INTRODUCTION}

Paper has always been the material basis for spreading civilization and culture (Yang 1999), in order to study the changes of mechanical and physical properties of some paper with the extension of storage time (Guo 2016). Garcia et al. (2019) prepared Cellulose pulp from the residual leaves of agave by alkali and acid methods. The results showed that the cellulose yield of acid pulping method was higher than that of alkaline pulping method. The paper obtained by alkaline pulping has lower humidity and higher tensile re- 
sistance. Tian et al. (2019) conducted dry heat aging tests on five kinds of paper, including bamboo paper and rice paper, in order to explore the preservation life of paper, the correlation coefficients of yellow return value, cold extraction $\mathrm{PH}$ value and tear index of four kind of paper with aging time were all above 0,9 showing very good correlation. Zhong et al. (2007) studied adding carbon fiber and other reagents into paper improve the tightness, tensile strength and breaking resistance of paper. To expand the understanding of the mechanical properties of paper before and after aging, Zhang (2017) used corn modified starch, sodium alginate, carboxymethyl cellulose three kinds of sizing agent to mix in proportion to kraft paper surface sizing, improved the mechanical properties of kraft paper. Kang et al. (2016) used nano-SiO hydrophobic coating on kraft paper and corrugated board improves the hydrophobic and physical properties of paper and cardboard to a certain extent. Chen et al. (2013) the dry strength of kraft paper was increased by replacing amphoteric starch with polyacrylamide reinforcing agent, and the effect of several polyacrylamide reinforcing agents was compared. Liu et al. (2018) prepared three different types of SKL copolymers. The results indicated that at a $2 \mathrm{wt} \%$ dosage of SKL copolymers, the increase in the physical properties of paper is maximum. Reis et al. (2011) coated Kraft paper with chitosan emulsion film Kraft paper was coated with chitosan emulsion film. Significantly reduced water vapor permeability rate and water absorption capacity. Thébault et al. (2018) proved that the ability of each resin to penetrate the kraft paper is related to the mechanical and chemical properties of the paper surface. Liu et al. (2018) made a simple underwater super - hydrophobic kraft paper net. Jimenez-Francisco et al. (2020) proved that kraft paper coated with rEPS/LL exhibits the highest tensile stiffness and compressive strength. Xu et al. (1999) compared two polymeric carboxylic acids, that is, PMA and PMMA for improving paper wet strength. Figueiredo et al. (2011) through the contact Angle measurement and surface energy analysis, it is concluded that the chemical composition and structure of kraft paper are the important factors determining the interaction with phenolic resin. Shankar et al. (2018) coated kraft paper with PBAT. The base weight and thickness of coated paper increased. The surface hydrophobicity and mechanical properties of coated paper are significantly improved compared with the original paper. Rhim et al. (2009) depending on the paper material, tensile strength of paper samples was affected by moisture content. Li et al. (2019) provided waterproof and oil-proof paper without plastic and fluorine, which is completely recyclable and has remarkable environmental benefits. Arifuzzaman et al. (2017) studied the effect of sodium silicate content in diluted adhesive on tensile properties of coated kraft paper. The results show that undiluted adhesive has the greatest influence on the strength and energy absorption of kraft paper. El-Sherif et al. (2018) modified kraft paper with high - efficiency synthetic adhesive and local pigment and its tensile strength and cracking strength were improved. El-Wakil et al. (2015) studied the effects of different proportions of $\mathrm{CNC}$ and $\mathrm{TiO}_{2}$ suspensions on tensile strength (TS), young's modulus and water sensitivity of kraft paper. To sum up, a large number of studies focused on increasing the mechanical properties and waterproof proper-ties of the paper, so as to increase the service life of the paper in the actual environment. However, the actual research on the paper with a longer storage time is less. In this study, kraft paper and watercolor paper before and after aging were taken as samples to analyze their practical significance.

Kraft paper and watercolor paper are used as the base materials in this experiment, and through the aging of paper to study the two kinds of paper in wetting, roughness, tensile tear and so on, the purpose of this study is to analyze the mechanical and physical properties of these two kinds of paper through scientific test methods, and to analyze whether kraft paper can replace watercolor paper in some aspects, so as to provide physical and chemical basis for the selection of painting and calligraphy paper.

\section{MATERIALS AND METHODS}

\section{Materials}

The model NO7146 is domestic all-wood pulp kraft paper and domestic Rubens watercolor paper, both of which are $300 \mathrm{~g} / \mathrm{m}^{2}$ and A4 paper $(210 \mathrm{~mm} \times 297 \mathrm{~mm})$. Application of watercolor powder (Faber-Castell, Germany) in wettability analysis of paper.

\section{Aging process}

In order to observe the changes of paper before and after aging, the MU3040C hot air aging machine (shanghai moujing industrial co. ltd), which was used to place kraft paper and watercolor paper in the clapboard layer of the aging chamber at $105^{\circ} \mathrm{C}$. the aging process was accelerated according to $\mathrm{GB} / \mathrm{T}+464-2008$ method $\mathrm{C}$ (treated at $105{ }^{\circ} \mathrm{C}$ for $72 \mathrm{~h}$ ), and the same amount of unaged kraft paper was stored in a dark place. The four kinds of paper before and after aging were marked with A, B, C and D (Table 1). 
Table 1: The annotation of paper.

\begin{tabular}{|c|cc|cc|}
\hline \multirow{3}{*}{ Sample } & \multicolumn{2}{|c|}{ Kraft paper } & \multicolumn{2}{c|}{ Watercolor paper } \\
\cline { 2 - 5 } Name & Before aging & After aging & Before aging & After aging \\
\cline { 2 - 5 } & A & B & C & D \\
\hline
\end{tabular}

\section{Solution preparation and infiltration angle analysis}

In order to study the wettability of watercolor paper and kraft paper before and after aging, the experiment selected distilled water as the matrix to label $\mathrm{S} 1$, and added $0,01 \mathrm{~g} 0,02 \mathrm{~g}$ and $0,03 \mathrm{~g}$ bulk watercolor dyes to $50 \mathrm{~mL}$ water solution by electronic balance. The mixed water color solution was labeled S2, S3 and S4 by ultrasonic dispersion for 20 minutes. The tag is shown in Figure 1. The wetting angle test of paper is to cut kraft paper and watercolor paper into $20 \mathrm{~mm} \times 60 \mathrm{~mm}$ rectangular strips at room temperature. The contact angle between kraft paper and watercolor paper is measured by using contact angle measuring instrument (Beijing Harco-SPCAX2 contact angle measuring instrument), the static contact angle of the two surfaces in the measured solution is $0,04 \mathrm{~mL}$ per drop of solution.

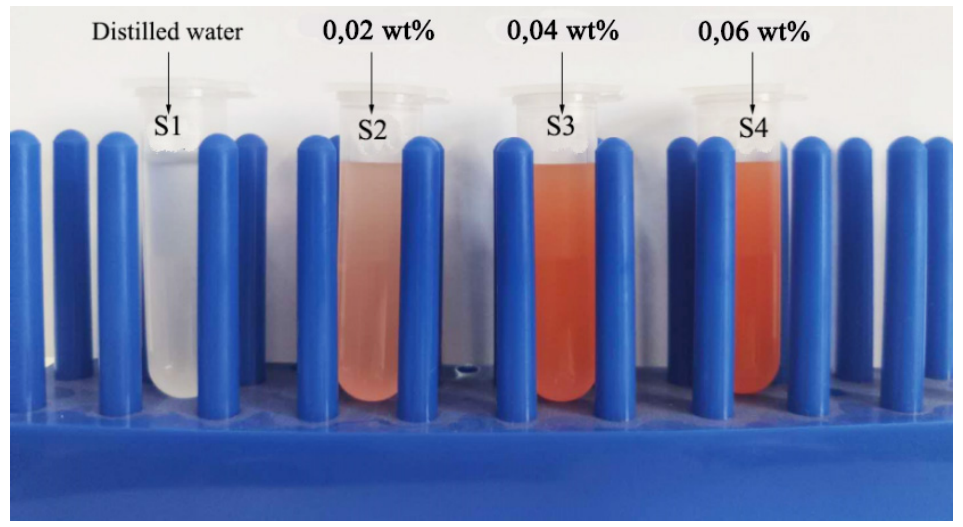

Figure 1: Mixing ratio of solution.

\section{Phase representation}

Four states of kraft paper and watercolor paper before and after aging were characterized by using Nicolet iS10 infrared spectrometer produced by Semerfer and D8 Advance powder X-ray diffractometer produced by Bruker, Germany. 2,5 Tensile tearing analysis and fracture characterization of paper

Tension and tearing of kraft paper and watercolor paper before and after aging were analyzed. Each sample was teared three times to reduce the error in the experiment. The SMT-5000 bending test machine produced by Yangzhou Saisi Testing Equipment Co., Ltd. was used. The maximum experimental force was $10 \mathrm{KN}$, the force precision was within $0,1 \%$, and the experimental speed was $0,1 \mathrm{~mm} / \mathrm{min}-500 \mathrm{~mm} / \mathrm{min}$. In order to understand the change of surface morphology before and after aging and to analyze the situation of paper after tensile tearing, scanning electron microscopy (FEI Quanta 65) was used. The tensile and tear fracture of the paper were compared and analyzed to observe the difference of the paper before and after aging. 
(a)

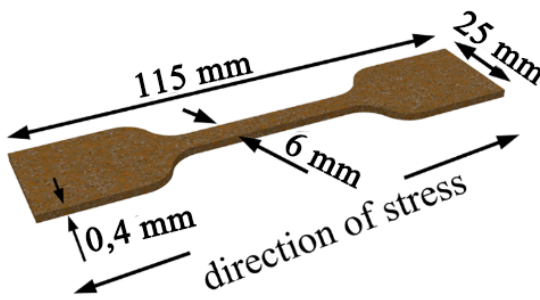

(b)

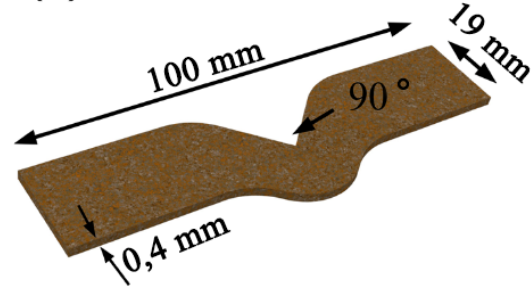

Figure 2: (a) The size specification of paper drawing, (b) The size specification of paper tear.

\section{Friction test}

XM-2A friction coefficient tester (Shanghai Zhonglu Industrial Co., Ltd.) was used to measure the surface roughness of watercolor paper and kraft paper before and after aging according to GB/T10006 method. Slip speed was controlled at $100+10 \mathrm{~mm} / \mathrm{min}$, weight was added on the basis of $200 \mathrm{~g}, 0 \mathrm{~g}, 20 \mathrm{~g}, 40 \mathrm{~g}, 60 \mathrm{~g}, 80 \mathrm{~g}$, $100 \mathrm{~g}$ to observe the two kinds of paper (Figure 3a). Variation of friction coefficient. In order to analyze the anti-friction performance of paper under actual environmental conditions, CFT-I multi-functional material surface comprehensive performance tester (Lanzhou Zhongke Kaihua Science and Technology Development Co., Ltd.) was used in the experiment (Figure 3b). The tester used reciprocating friction form, load $10 \mathrm{~N}$, running speed $100 \mathrm{rpm} / \mathrm{min}$, running length $5 \mathrm{~mm}$, and NANOVEA ST400 three-dimensional topography tester. The wear morphology after friction was analyzed. The flow charts of the two friction experimental principles are shown in Figure 2.

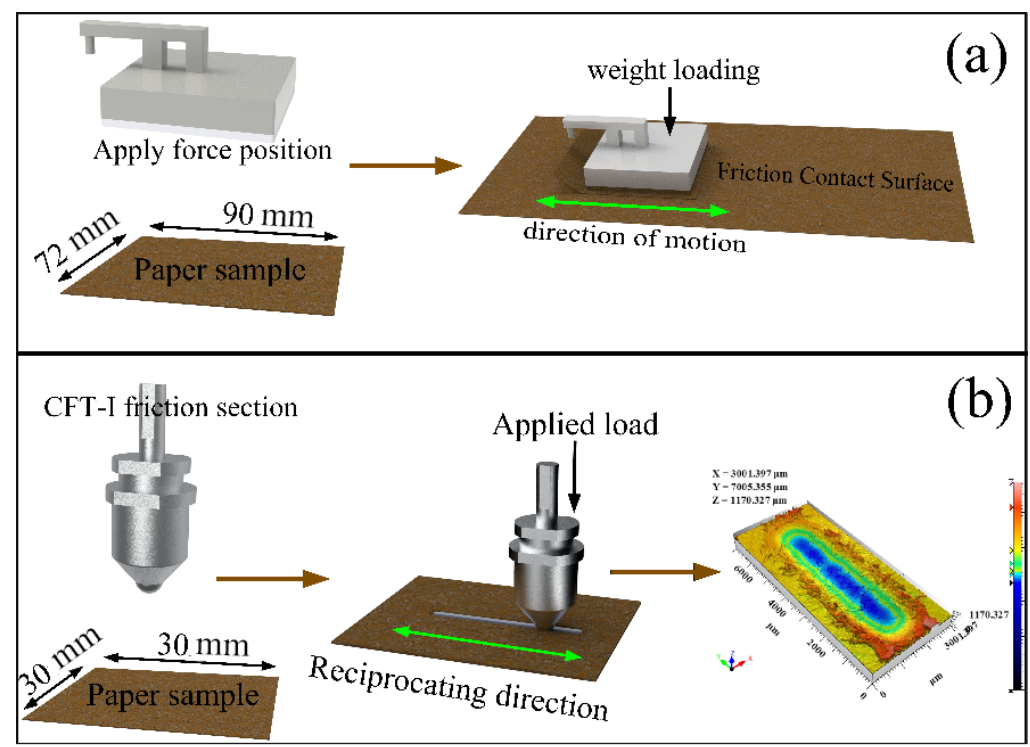

Figure 3: Friction diagram of Two Kinds of Paper, (a) dynamic and static friction process. (b) flow chart of friction for dual pairs. 


\section{RESULT AND DISCUSSION}

\section{Characterization results of paper}

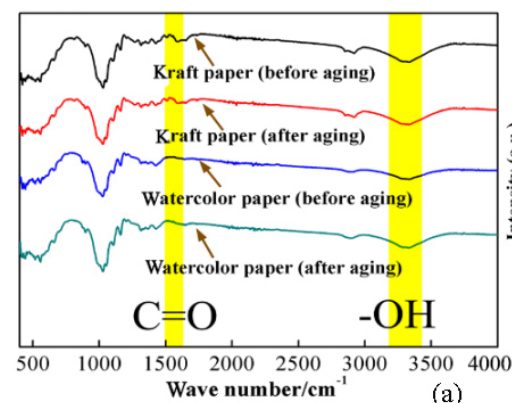

(a)
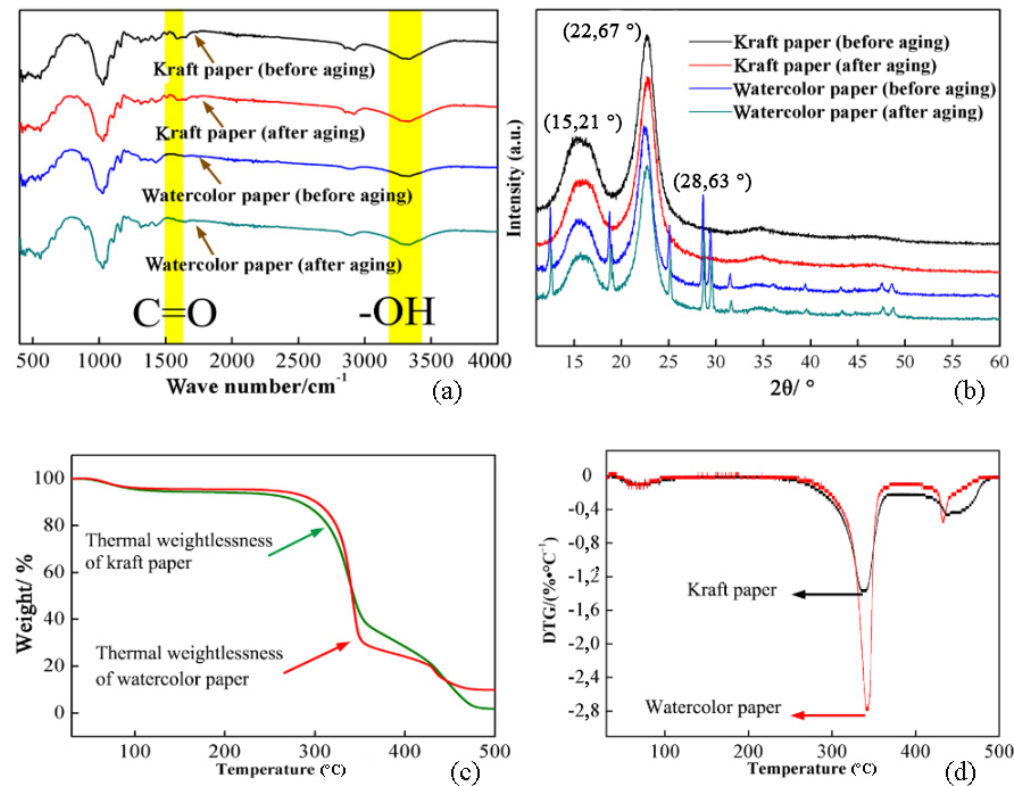

Figure 4: Characterization of two kinds of paper before and after aging, (a) characterized by infrared spectroscopy, (b) the representation of XRD, (c) the thermogravimetric analysis of paper, (d) the DTG (differential thermogravimetric) curve of two kinds of paper.

As shown in Figure 4a represents the infrared spectra of four kinds of paper. It can be seen from the figure that the position of functional groups of kraft paper and watercolor paper does not change seriously before and after aging. It can be seen that $\mathrm{C}=\mathrm{O}$ group exists in $1735 \mathrm{~cm}^{-1}$ and $-\mathrm{OH}$ group exists in $3300 \mathrm{~cm}^{-1}$; The molecular vibration of kraft paper - OH group near is greater than the watercolor paper; Figure $4 \mathrm{~b}$ shows that there are no obvious diffraction peaks in the range of diffraction angle before and after aging of kraft paper, and there are two obvious "steamed bread peaks" between $10^{\circ}$ and $30^{\circ}$, it shows that kraft paper is amorphous; before and after aging of watercolor paper, there are more obvious diffraction peaks except «steamed bread peak», which can be seen from the analysis. Small-angle diffraction peaks are caused by the porous structure of paper. Figure $4 \mathrm{c}$ and Figure $4 \mathrm{~d}$ are the thermogravimetric analysis of two kinds of papers. From Figure $4 \mathrm{c}$, it can be seen that the quality of watercolor paper and kraft paper has little change before $280^{\circ} \mathrm{C}$, mainly in the stage of water loss. With the further increase of temperature, the thermogravimetric analysis of the two kinds of papers is most obvious in the temperature range of $280^{\circ} \mathrm{C}-350^{\circ} \mathrm{C}$, and the weight loss rate of watercolor paper is faster than kraft paper. The results show that the maximum weight loss rate of watercolor paper and kraft paper is about $350^{\circ} \mathrm{C}$, which also proves that the weight loss rate of watercolor paper is higher than kraft paper.

From Figure 5, we can see the water contact angle of the same solution in four states. From Figure 5a, we can see that the contact angle of the watercolor paper before and after aging belongs to the hydrophobic region. The contact angle values are $117,86^{\circ}$ and $111,10^{\circ}$, however, the contact angle of the kraft paper before aging is $74,66^{\circ}$, showing hydrophilicity, while the contact angle after aging is $117,86^{\circ}$ and $111,10^{\circ}$, respectively. The antenna is $92,52^{\circ}$, showing hydrophobicity, but the hydrophobicity is inferior to watercolor paper; the contact angle measured in S2 solution (Figure 5b), The contact angle of kraft paper before and after aging is $79,34^{\circ}$, which belongs to hydrophilic material. The contact angle of kraft paper after aging is $91,99^{\circ}$, which shows hydrophobicity. It can be seen from Figure $5 \mathrm{c}$ and Figure $5 \mathrm{~d}$ that the contact of kraft paper measured in solution S3 and S4 is hydrophobic. The contact angle of the watercolor paper before and after aging is between $114^{\circ}$ 
and $118^{\circ}$ and it is still hydrophobic. The kraft paper before and after aging is hydrophilic in both solutions, and the contact angle is between $71^{\circ}$ and $81^{\circ}$.

It can be seen that watercolor paper shows hydrophobicity in four kinds of watercolor solutions, the contact angle changes little before and after aging, which indicates that watercolor paper has stronger anti-aging ability, while kraft paper shows different properties before and after aging in the comparison of contact angle between distilled water and S1 solution, and it shows hydrophilicity before aging. As the concentration of watercolor solution increases, kraft paper shows hydrophilicity gradually before and after aging. The aging kraft paper shows hydrophobic and hydrophilic color dye characteristics, and also shows that kraft paper has poor anti-aging performance.
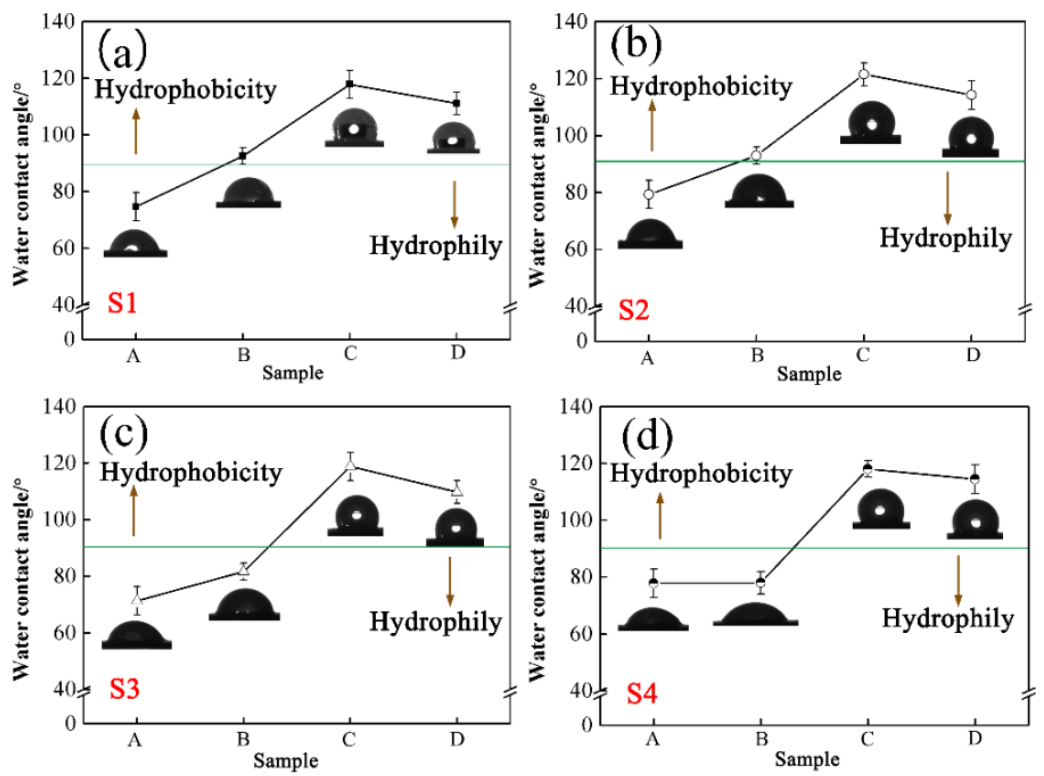

Figure 5: Contact angles of paper in four dye solutions, (a) the contact angle of S1 pair of paper, (b) the contact angle of S2 pair of paper, (c) the contact angle of S3 pair of paper, (d) ithe contact angle of S4 pair of paper.

The change of contact angle of paper can be explained by surface shape and dynamic and static friction coefficient (Figure 6). The surface morphology of kraft paper changed obviously before and after aging. The interfibre bonding changed from single bonding to flaky area bonding. The hairy substance of kraft paper surface increased after aging. From the analysis of dynamic and static friction coefficient, the surface friction coefficient of kraft paper after aging was larger than kraft paper before aging, and the surface roughness was higher than kraft paper before aging. The increase of contact angle causes the change of contact angle, and the composition of kraft paper is composed of cellulose, resin and other lipids. Resins contain strong polar functional groups. They have a great affinity for water and promote the absorption of solution. The moisture content of kraft tissue decreases after aging, and the internal sizing degree increases, which improves the water resistance of paper. but the contact angle of kraft paper decreases with the increase of dye solution. Observation of SEM images before and after aging of watercolor paper shows differences in the surface, but the difference is not very big. The surface roughness of watercolor paper becomes larger after aging. This is the paper scraps produced on the surface after aging. The processing technology of watercolor paper is different from kraft paper. The surface hydrophobicity is consistent with the purpose of using. The fiber of watercolor paper is small. The smaller interstitial pore makes the wetting angle larger, and the contact angle of watercolor paper in four solutions is roughly the same, indicating that the hydrophobicity of watercolor paper before and after aging does not change with the change of solution concentration. 


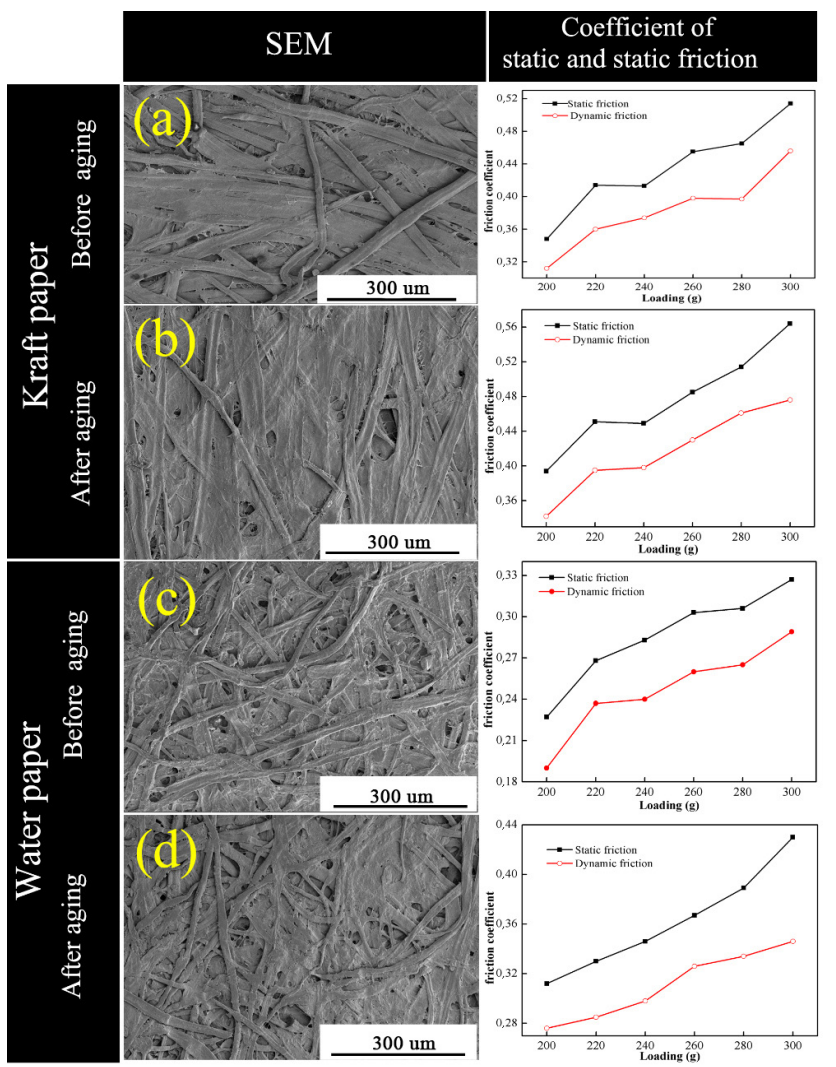

Figure 6: Dynamic and Static Friction Coefficients of Surface SEM and Surface of Paper, (a) denotes the surface of kraft paper before aging, (b) denotes the surface of kraft paper after aging, (c) denotes the surface of watercolor paper before aging, (d) denotes the surface of watercolor paper after aging.

\section{Tensile tearing analysis of paper}

In order to understand the mechanical differences and advantages and disadvantages between kraft paper and watercolor paper, the tensile and tearing tests of two kinds of paper before and after aging were carried out. Three times of tests were repeated for each kind of paper to reduce the existence of test errors. The tensile and tearing data of the samples were the average values obtained by three times of each sample test.
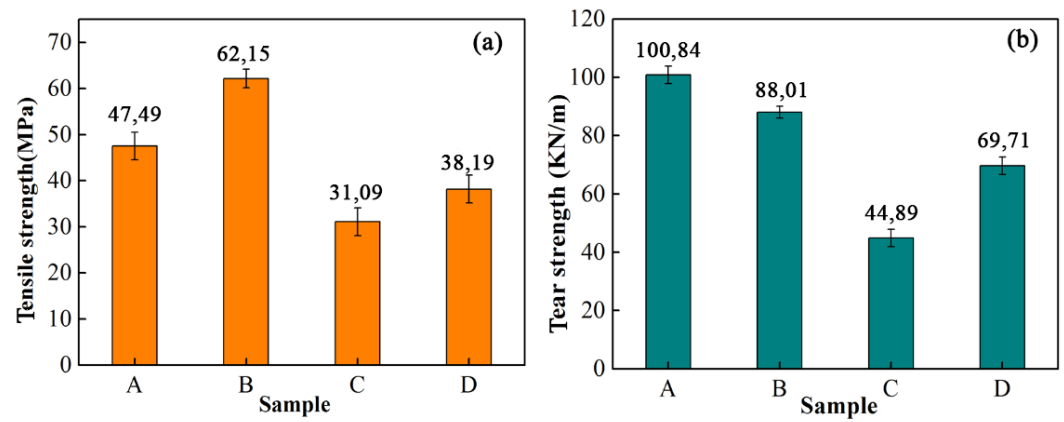

Figure 7: (a) the tensile strength of paper, (b) the tear strength of paper. 
In order to analyze the stress state of the specimens after tension and tearing more clearly, the SEM photographs of the fracture surface of the specimens after tension and tearing were taken to facilitate further understanding of the fiber state at the fracture surface (Figure 8).

From Figure 7a, it can be seen that the tensile strength of kraft paper before and after aging is much higher than watercolor paper. The tensile strength of kraft paper after aging is the best. The tensile strength of kraft paper before aging is $47,49 \mathrm{MPa}$, and kraft paper after aging is $62,15 \mathrm{MPa}$, However, watercolor paper shows poor tensile strength, The tensile strength of watercolor paper before aging is $31,09 \mathrm{MPa}$, while watercolor paper after aging is $38,19 \mathrm{MPa}$. For manufacturing purposes, the tensile strength of kraft paper is higher than watercolor paper. From Figure 6, it can also be observed that kraft paper has larger and longer fibers. Watercolor paper has smaller fibers, and larger fibers increase the toughness of the paper. The tensile strength of kraft paper is greater than watercolor paper. Compared with the tensile strength of the same kind of paper before and after aging, the tensile strength of kraft paper after aging is the best. From the surface shape of the paper in Figure 6, it can be seen that the single fiber of kraft paper decreases, the porosity decreases, the connection between the fibers is tight, and the sizing content of kraft paper increases after aging, which increases the tensile strength of kraft paper. From the fracture of kraft paper in Figure 8 was described a,e,b,f for before and after aging, it can be seen that there are more fiber fracture after aging, less fiber fracture before aging, and most of them are the whole shedding of fibers. The tensile strength of watercolor paper after aging is also greater than that before aging. From the fracture of $\mathrm{c}$ and $\mathrm{d}$ watercolor paper in Figure 8, it can be seen that the watercolor paper after aging is watercolor paper after aging. There are obvious fiber fracture layers, and the breakage of watercolor paper fibers before aging coexists with single fiber shedding, which shows that the bonding strength between the fibers of watercolor paper after aging is larger, so the tensile strength of the aged kraft paper is greater than that before aging, and the aged watercolor paper is better than that before aging, but overall comparison, kraft paper is better than before aging. The tensile strength of kraft paper is better than watercolor paper, and kraft paper is the best after aging.

From Figure $7 \mathrm{~b}$, the tearing strength of kraft paper is better than watercolor paper. The tearing strength of kraft paper before aging is $100,84 \mathrm{kN} / \mathrm{m}$, and kraft paper after aging is $88,01 \mathrm{kN} / \mathrm{m}$. The tearing strength of watercolor paper is worse than kraft paper. The tearing strength of watercolor paper before aging is $44,89 \mathrm{kN} / \mathrm{m}$, and watercolor paper after aging is $88,01 \mathrm{kN} / \mathrm{m}$. The tear strength of watercolor paper is $69,71 \mathrm{kN} / \mathrm{m}$. The tear strength of watercolor paper is much smaller than kraft paper. The kraft paper has longer and thicker fibers and better tear resistance. The surface shape of kraft paper and watercolor paper observed from Figure 6 shows that there are more squares before aging, more squares before aging, and more plain weaves for watercolor paper, while the tear resistance of squares fabric is much higher than plain weave fabric, but the tear strength of kraft paper after aging is worse than that before aging.

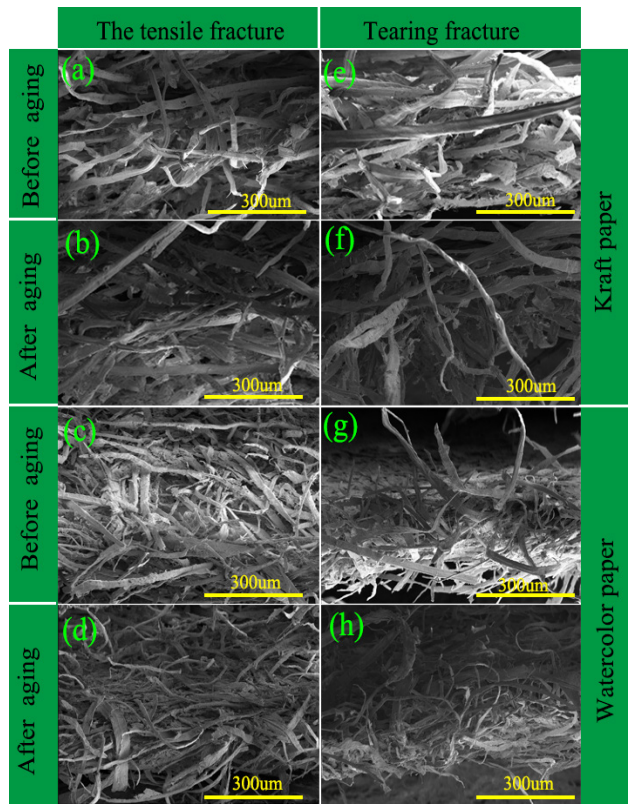

Figure 8: (a) Tensile and (e) tear fracture of kraft paper before aging, (b) and (f) tensile and tear fracture of kraft paper after aging, (c) and (g) tensile and tear fracture of watercolor paper before aging, (d) and (h) tensile and tear fracture of aged watercolor paper. 
From the fracture of Figure 8e and Figure 8f, it can be seen that the kraft paper fibers break more before aging. It shows that the transverse force of the kraft paper fibers is greater when they are teared, while the fiber breaks off obviously after aging. It shows that the kraft paper changes the fabric state after aging, and the tear resistance of the kraft paper fibers decreases. The tear strength of the paper is better than the paper before aging as shown in Figure $7 \mathrm{~b}$. From the Figure $8 \mathrm{~g}$ and Figure $8 \mathrm{~h}$, it can be seen that the fibers of watercolor paper before aging fall off seriously during tearing, which indicates that the fibers' binding force is not very strong when they are teared transversely. After tearing, the fibers of watercolor paper after aging are severely broken, which indicates that the fibers receive the "resistance" of the interfibers" binding force when they are teared transversely, which proves that watercolor paper has a strong resistance to the interfibers' binding force. After aged, the bonding strength between fibers changes.

\section{Friction analysis of paper}

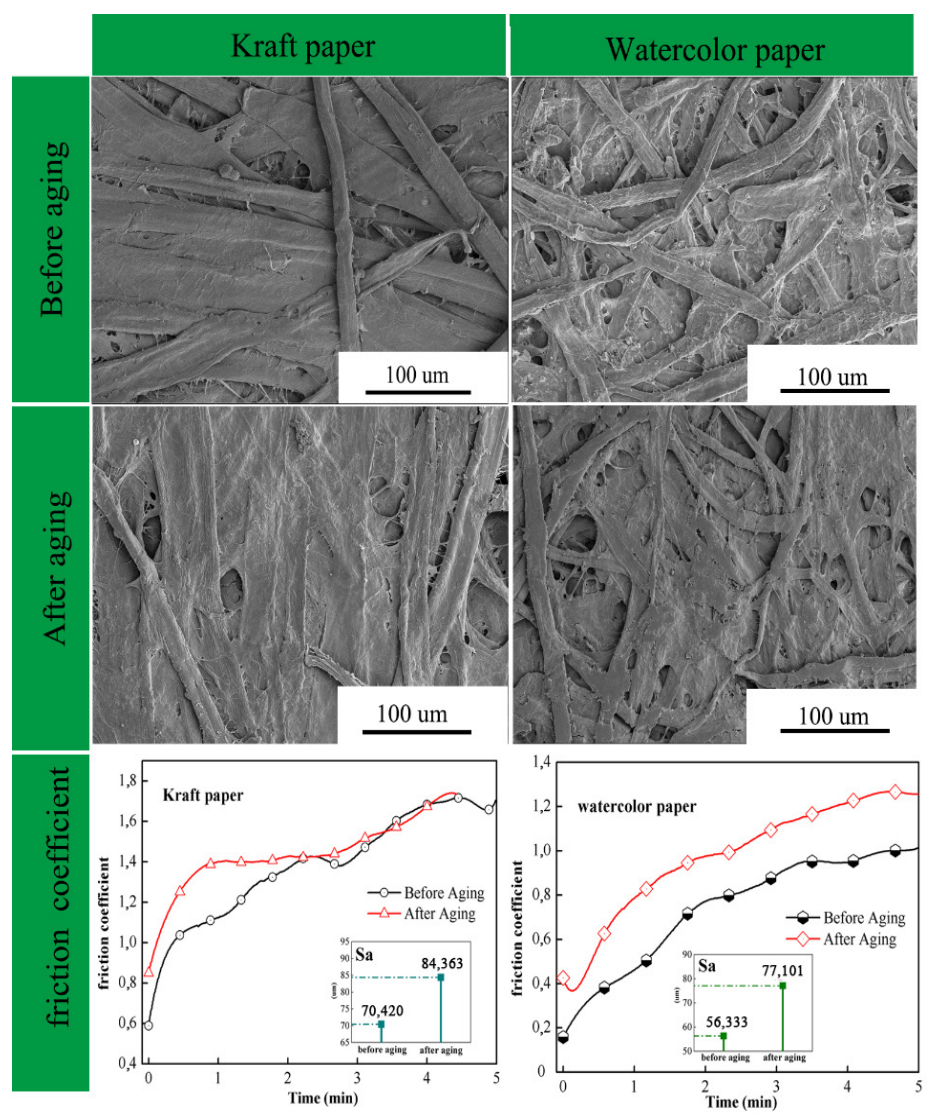

Figure 9: Variation of Surface Friction Coefficient of Two Kinds of Paper.

In order to analyze the surface state of two kinds of paper before and after aging after external friction, the steel ball with dual pair GCr15 was used for friction analysis. In the above friction environment, the surface friction coefficient of kraft paper and watercolor paper before and after aging could be obtained by reciprocating friction on the surface of kraft paper and watercolor paper. The surface arithmetic square root Sa is obtained from the three-dimensional topography of the frictional part of the surface Figure 9.

From the above figure, we can see that the friction coefficient of kraft paper before and after aging is higher than watercolor paper. From the analysis of paper surface, the surface smoothness of watercolor paper is much better than kraft paper. From the SEM chart of paper, we can also see that the fiber of kraft paper is thicker, the fiber burrs are more, and the fiber of watercolor paper is much larger. The friction coefficient of kraft paper is larger than watercolor paper. 
The friction coefficient of kraft paper after aging is larger than before aging, and gradually tends to 1,6. The change of friction coefficient is related to the morphology of fiber, bonding strength between interfaces, hot pressing pressure, porosity and size. The SEM images of kraft paper before and after aging show that the morphology of fiber changes obviously, and the fiber after aging. When the paper is aged, the dehydration is serious, the compressive modulus of elasticity is low and the friction coefficient is high. After a period of friction, the friction environment of the two tends to be the same and the friction coefficient tends to be the same. The friction coefficient of watercolor paper before aging is also less than that after aging. The friction coefficient before aging is 0,9 and after aging is 1,2 . From the SEM images of both, it can be seen that there are more fiber debris on the surface of watercolor paper after aging, the friction environment is deteriorating, and the water loss of the fiber after aging is serious, from the friction part. The environmental heat dissipation and local compression are considered as elastic modulus. The friction coefficient of watercolor paper after aging is larger than that before aging. As can be seen from the figure above, under the same environment, the Sa value of kraft paper is larger than watercolor paper.

In Figure 10, from the three-dimensional morphology of the frictional parts of the four kinds of paper shown above, we can see that the average depth of kraft paper before aging is less than kraft paper. The average depth of kraft paper is $191,622 \mu \mathrm{m}$, and the average depth of watercolor paper is $238,645 \mu \mathrm{m}$ (figure 10a and 10c). This indicates that kraft paper is in the same environment before aging. The average friction depth of kraft paper after aging is $242,909 \mu \mathrm{m}$, while watercolor paper after aging is $235,421 \mathrm{um}$ (figure $10 \mathrm{~b}$ and $10 \mathrm{~d}$ ), but the lowest depth of kraft paper is less than watercolor paper, which shows that kraft paper is still more wear-resistant than watercolor painting after aging.
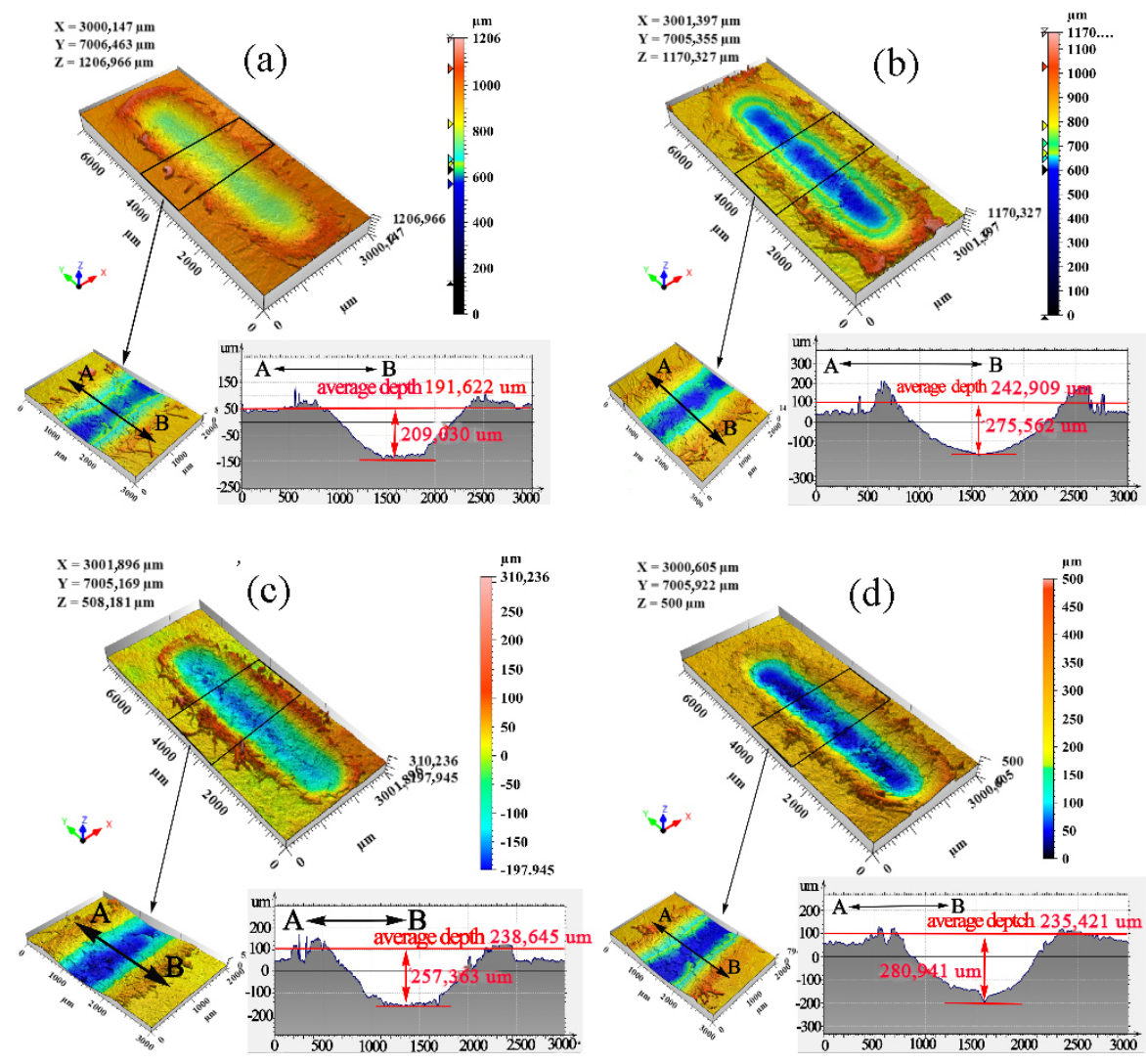

Figure 10: (a)The three-dimensional morphology of kraft paper after friction before aging, (b) is the three-dimensional morphology of kraft paper after aging and friction, (c) is the three-dimensional morphology of watercolor paper after friction before aging, (d) is the three-dimensional morphology of watercolor paper after aging and friction. 
Comparing the friction state of kraft paper before and after aging, it can be seen that the friction depth before aging is smaller than after aging, the friction coefficient increases after aging, the flexibility of fibers decreases, and the surface state of paper changes, all of which make the wear resistance of kraft paper weaken; watercolor paper is also better than before aging, after aging. Wear resistance reduction can still be understood from the analysis of kraft paper before and after aging.

\section{CONCLUSIONS}

In the experiment of contact angle between distilled water and three different concentration of water color solution, kraft paper before aging showed hydrophilicity. The contact angle of aging kraft paper in distilled water was $92,52^{\circ}$. The aging kraft paper showed hydrophobicity. For water color paper, the watercolor paper before and after aging showed hydrophobic. Compared with the contact angle, the contact angle of kraft paper after aging is smaller, but it also shows hydrophobic properties. This can be explained by the surface properties of the tested paper. The aging test has serious damage to the kraft paper surface, poor aging resistance, and less damage to the watercolor paper.

In the aspect of tensile and tearing, kraft paper shows better tensile and tearing resistance. In the aspect of tensile strength, the tensile strength of aged kraft paper reaches $62,15 \mathrm{MPa}$, while watercolor paper shows poor tensile performance. The tensile strength of aged watercolor paper is $38,19 \mathrm{MPa}$, and the aged kraft paper is the best in tearing. In terms of tear strength, the tear strength of kraft paper after aging is $88,01 \mathrm{kN} / \mathrm{m}$, and the tear strength of watercolor paper is worse than kraft paper. The tear strength of watercolor paper after aging is $69,71 \mathrm{kN} / \mathrm{m}$. The aging kraft paper shows better performance than watercolor paper.

The friction coefficient curve of kraft paper before and after aging tends to be stable at 1,9, while watercolor paper tends to be stable at 0,9 and watercolor paper tends to be stable at 1,2 after aging. The friction coefficient of the two kinds of paper is bigger than the watercolor paper, and the surface friction coefficient of the two kinds of paper is bigger after aging. From the three-dimensional morphology images, it can be seen that the kraft paper before aging shows better wear resistance than the watercolor paper before aging. However, the friction surface of the two kinds of paper becomes worse after aging, and the friction depth increases, thus affecting the wear resistance of the two kinds of paper.

\section{ACKNOWLEDGEMENTS}

This research project was supported by the scientific and technological innovation talents of universities in Henan Province (19HASTIT023).

\section{REFERENCES}

Arifuzzaman, M.; Kim, H.S. 2017. Novel flexural behaviour of sandwich structures made of perlite foam/sodium silicate core and paper skin. Constr Build Mater 148: 321-333. http://doi.org/10.1016/j.conbuildmat.2017.05.073

Chen, Y.L.; Zhou, J.H.; Sun, J.D. 2013. Application of polyacrylamide dry - strengthening agent in kraft paper production. Hunan Papermak 32(2): 21-22.

El-Sherif, H.M.; Nasser, A.M.; Hussin, A.I. 2018. Tailoring of mechanical properties and printability of coated recycled papers. Poly Bull 76: 2965-2990. http://doi.org/10.1007/s00289-018-2515-7

El-Wakil, N.; Hassan, A.E.; Abou-Zeid, A.R.E. 2015. Development of wheat gluten/nanocellulose/titanium dioxide nanocomposites for active food packaging. Carbohydr Poly 124: 337-346. http://doi.org/10.1016/j.carbpol.2015.01.076

Figueiredo, A.B.; Evtuguin, D.; Monteiro, V.J. 2011. Structure-Surface Property Relationships of Kraft Papers: Implication on Impregnation with Phenol-Formaldehyde Resin. Industr Eng Chem Res 50(5): 2883- 
2890. http://doi.org/10.1021/ie101912h

Guo, Y.N. 2016. Comprehensive study on mechanical properties of artificially aged paper. $J$ Liaoning Police Col (1): 81-84.

Garcia, F.P.; Muñoz, E.J.; Sandoval, O.A.A. 2019. Obtaining and Optimization of Cellulose Pulp from Leaves of Agave tequilana Weber Var. Blue. Preparation of Handmade Craft Paper. Waste Bio 10(8): 23792395. http://doi.org/10.1007/s12649-018-0262-5

Jimenez-Francisco, M.; Flores-Johnson, E.A.; Carrillo, J.G. 2020. Effect of Recycled Polystyrene/ Limonene Coating on the Mechanical Properties of Kraft Paper: A Comparative Study with Commercial Coatings. J Poly Environ 28: 1724-1736. http://doi.org/10.1007/s10924-020-01721-y

Kang, L.; Huan, Y.; Mu, J. 2016. Effect of nano- $\mathrm{SiO}_{2}$ hydrophobic coating on the properties of paper and paperboard. Packag Eng 37(9): 21-25.

Liu, Z.M.; Xu, D.D.; Xu, L. 2018. Preparation and Characterization of Softwood Kraft Lignin Copolymers as a Paper Strength Additive. Polymers 10: 743-755. http://doi.org/10.3390/polym10070743

Liu, Y.Q.; Jiao, Z.Z.; Zhang, Y.L. 2018. Kraft Mesh Origami for Efficient Oil-Water Separation. Langmuir 12: 1-30. http://doi.org/10.1021/acs.langmuir.8b03541

Li, Z.; Rabnawaz, M.; Sarwar, M.G. 2019. A closed-loop and sustainable approach for the fabrication of plastic-free oil- and water-resistant paper products. Green Chem 21: 5691-5700. http://doi.org/10.1039/c9gc01865d

Reis, A.B.; Yoshida, C.M.; Reis, A.P.C. 2011. Application of chitosan emulsion as a coating on Kraft paper. Poly Int 60(6): 963-969. https://doi.org/10.1002/pi.3023

Rhim, J.W.; Lee, J.H. 2009. Thermodynamic Analysis of Water Vapor Sorption Isotherms and Mechanical Properties of Selected Paper-Based Food Packaging Materials. J Food Sci 74(9): 502-511. http://doi.org/10.1111/j.1750-3841.2009.01373.x

Shankar, S.; Rhim, J.W. 2018. Effects of poly(butylene adipate-co-terephthalate) coating on the water resistant, mechanical, and antibacterial properties of Kraft paper. Prog Org Coat 123: 153-159. http://doi.org/10.1016/j.porgcoat.2018.07.002

Tian, Z.L.; Zhang, M.; Ren, S.S. 2019. Statistical analysis of physical and chemical indexes and aging time of paper. Cult Relic Prot Archaeological Sci 30 (1): 67-73.

Thébault, M.; Kandelbauer, A.; Zikulnig-Rusch, E. 2018. Impact of phenolic resin preparation on its properties and its penetration behavior in Kraft paper. European Polymer Journal 104: 90-98. http://doi.org/10.1016/j.eurpolymj.2018.05.003

Xu, G.G.; Yang, Q.X. 1999. Comparison of the kraft paper crosslinked by polymeric carboxylic acids of large and small molecular sizes. Journal of Applied Polymer Science 74(4): 907-912. https://doi.org/10.1002/ (SICI)1097-4628(19991024)74:4<907::AID-APP17>3.0.CO;2-9

Yang, M.S. 1999. Prospect of sustainable development of Chinese paper industry. China Paper Indust (1): $9-11$

Zhong, L.X.; Zhang, M.Y.; Liu, Z.W. 2007. Characteristics of carbon fiber and its application in functional paper. China Papermak 26(11). 51-54

Zhang, R. 2017. Effect of sizing components on mechanical properties of kraft paper. Print Fie 46(11): 